\title{
Relativistic theory for time and frequency transfer to order $c^{-3}$
}

\author{
L. Blanchet ${ }^{1}$, C. Salomon ${ }^{2}$, P. Teyssandier ${ }^{3}$, and P. Wolf ${ }^{4}$ \\ 1 Département d'Astrophysique Relativiste et de Cosmologie, Observatoire de Paris, 92195 Meudon Cedex, France \\ 2 Laboratoire Kastler Brossel, École Normale Supérieure, 24 rue Lhomond, 75231 Paris, France \\ 3 Observatoire de Paris, DANOF, CNRS/UMR 8630, 61 avenue de l'Observatoire, 75014 Paris, France \\ 4 Bureau International des Poids et Mesures, Pavillon de Breteuil, 92312 Sèvres Cedex, France
}

Received 2 November 2000 / Accepted 2 February 2001

\begin{abstract}
This paper is motivated by the current development of several space missions (e.g. ACES on International Space Station) that will use Earth-orbit laser cooled atomic clocks, providing a time-keeping accuracy of the order of $510^{-17}$ in fractional frequency. We show that to such accuracy, the theory of frequency transfer between Earth and Space must be extended from the currently known relativistic order $1 / c^{2}$ (which has been needed in previous space experiments such as GP-A) to the next relativistic correction of order $1 / c^{3}$. We find that the frequency transfer includes the first and second-order Doppler contributions, the Einstein gravitational red-shift and, at the order $1 / c^{3}$, a mixture of these effects. As for the time transfer, it contains the standard Shapiro time delay, and we present an expression also including the first and second-order Sagnac corrections. Higher-order relativistic corrections, at least $\mathcal{O}\left(1 / c^{4}\right)$, are numerically negligible for time and frequency transfers in these experiments, being for instance of order $10^{-20}$ in fractional frequency. Particular attention is paid to the problem of the frequency transfer in the two-way experimental configuration. In this case we find a simple theoretical expression which extends the previous formula (Vessot et al. 1980) to the next order $1 / c^{3}$. In the Appendix we present the detailed proofs of all the formulas which will be needed in such experiments.
\end{abstract}

Key words. relativity - reference systems - time

\section{Introduction}

Recent advances in laser cooling of atoms have led to the development of a number of highly accurate atomic clocks (Caesium and Rubidium fountains) which have improved time-keeping accuracy by an order of magnitude during the nineties to currently $\approx 10^{-15}$ in fractional frequency (Laurent et al. 1999; Bize et al. 1999). For further improvement of accuracy, the fountain clocks are limited by gravity, and therefore several experiments are planned for the near future that will fly laser cooled atomic clocks on board terrestrial satellites. One of these is ESA's Atomic Clock Ensemble in Space (ACES) mission (Salomon \& Veillet 1996) planned for 2005. This mission will place a laser cooled caesium clock together with a hydrogen maser on board the International Space Station. When combined with equally accurate time/frequency transfer systems that allow the comparison between space and ground clocks, the experiment should become a useful tool for a number of applications in metrology, fundamental physics, atmospheric studies, geodesy etc.

Send offprint requests to: L. Blanchet, e-mail: Luc.Blanchet@obspm.fr
Therefore the ACES mission will include sufficiently stable optical and microwave time and frequency transfer systems to allow the comparison of the clocks with negligible noise contribution from the transfer system itself. At the required accuracy this condition calls for two-way systems that exchange electromagnetic pulses in two directions in order to eliminate or reduce a number of unwanted effects associated with the instrumental delays, the propagation within the ionosphere and troposphere, etc. The Time Transfer by Laser Light (T2L2) system (Fridelance et al. 1996) uses optical pulses that are emitted on the ground, reflected by the satellite and received back on the ground. The events of emission and reception are dated on the ground clock, and the event of reflection on the satellite clock. The microwave system is expected to exchange pulses in both directions and date all events of emission and reception. It will also measure the frequency of the emitted and received pulses on board and on the ground. Additionally it is expected to include two downlink signals at different frequencies in order to allow the cancellation of the residual ionospheric effect.

At the required uncertainties a fundamentally relativistic modelling of the experiment is indispensable. 
Indeed the relativistic effects when comparing two clocks separated by an altitude of $\approx 400 \mathrm{~km}$ (as is the case for ACES) can amount to several parts in $10^{-11}$, which exceeds the expected uncertainties of the clocks $\left(\approx 510^{-17}\right)$ by several orders of magnitude. Evidently, a relativistic treatment of distant clock comparisons using electromagnetic signals becomes necessary whenever the uncertainties of such experiments become smaller than the size of the relativistic corrections. This has already been the case for the gravity probe A (GP-A) experiment (Vessot et al. $1980)$, and is more generally the case since the advent of the Global Positioning System (GPS) which is now extensively used for distant clock comparisons.

The first theoretical treatments of such comparisons in a relativistic framework were performed by Jaffe \& Vessot (1976), Ashby \& Allan (1979), Vessot et al. (1980), Allan \& Ashby (1985), and Klioner (1993). Subsequent new methods with improved uncertainties, such as the Two Way Time Transfer (TWTT) (Hetzel \& Soring 1993) and the Laser Synchronization from Stationary Orbit (LASSO) (Veillet \& Fridelance 1993), have led to the need for more accurate theoretical treatments that include some higher order terms (Petit \& Wolf 1994, 1997; Wolf \& Petit 1995; Klioner \& Fukushima 1994). The latter papers treat in some detail the synchronization (time transfer) between distant clocks using electromagnetic signals and the relation between the proper time of a clock on the Earth or on board terrestrial satellites and the geocentric coordinate time, TCG. The relativistic theory for frequency transfer has been revisited recently by Ashby (1998), leading to results similar to those presented in Sect. 4.2. In the perspective of the expected uncertainties of the ACES and other similar experiments, for time as well as frequency transfer, a re-examination of these formalisms has proved necessary. This is the subject of the present paper.

Essentially we shall compute the relativistic transfers of time and frequency, including all the terms up to the order $1 / c^{3}$. The coordinate time transfer up to this order is well known as it consists of the standard Shapiro time delay. Concerning the frequency transfer, we find that the formula for the one-way transfer is rather complicated. Our main result will concern the formula for the twoway frequency transfer, up to order $1 / c^{3}$. This formula appeared previously in the paper by Ashby (1998) but without a detailed derivation. In this paper, we derive this formula and, more generally, we present a self-contained derivation of all the formulae needed in this context. These formulae will be of direct use in the ACES experiment, and a fortiori in more precise future experiments. Higherorder relativistic corrections are negligible with respect to the projected uncertainties associated with ACES.

The numerical applications made in this paper concern the ACES mission with a transfer from the Space Station A orbiting at the altitude $H=400 \mathrm{~km}$ to a ground station located at B. For the velocities involved we use $v_{\mathrm{A}}=7.710^{3} \mathrm{~m} / \mathrm{s}$ and $v_{\mathrm{B}}=v_{\text {ground }}=465 \mathrm{~m} / \mathrm{s}$; for the gravitational potentials, $U_{\mathrm{B}} / c^{2}=6.910^{-10}$ and $U_{\mathrm{A}} / c^{2}=6.510^{-10}$; and for the Earth parameters
$G M_{\mathrm{E}}=3.9810^{14} \mathrm{~m}^{3} / \mathrm{s}^{2}$ and $R_{\mathrm{E}}=6.3710^{6} \mathrm{~m}$. We consider that the experimental uncertainties of ACES will be at the level of 5 ps for time transfer and $510^{-17}$ for frequency transfer.

The paper is organized in such a way that the main text summarizes all the results needed by an experimental team in setting up a relativistic time and/or frequency transfer, with all the proofs and theoretical details relegated to the Appendix. In Sect. 2 we treat the problem of the transformation from proper time to coordinate time TCG at a level sufficient for ground clocks and the ACES space clock. In Sect. 3 we give the expressions required for time transfer (including Sagnac terms), and in Sect. 4 for frequency transfer. Both the one-way and two-way transfers are considered in each case.

\section{Proper time in terms of coordinate time}

Throughout this work we use the geocentric inertial (nonrotating) coordinate frame GRS: Geocentric Reference System. Thus, $x^{0} / c=t=$ TCG is the geocentric coordinate time ${ }^{1}$, and $\boldsymbol{x}=\left(x^{i}\right)$ are the GRS harmonic spatial coordinates, for which the spatial metric is conformally flat to order $1 / c^{2}$. In these coordinates the metric interval including all the terms up to the order $1 / c^{2}$ reads

$$
\begin{aligned}
\mathrm{d} s^{2} & \equiv-c^{2} \mathrm{~d} \tau^{2} \\
& =-\left(1-\frac{2 U}{c^{2}}\right) c^{2} \mathrm{~d} t^{2}+\left(1+\frac{2 U}{c^{2}}\right) \delta_{i j} \mathrm{~d} x^{i} \mathrm{~d} x^{j}
\end{aligned}
$$

where $U$ is the total Newtonian potential, with the convention that $U \geq 0$ (IAU 1991), and where $\delta_{i j} \mathrm{~d} x^{i} \mathrm{~d} x^{j}=\mathrm{d} \boldsymbol{x}^{2}$ denotes the Euclidean space metric. The Solar-system barycentric coordinates, centred on the barycenter of the Solar system, are denoted $\mathcal{T}=$ TCB: solar system barycentric coordinate time, and $\mathcal{X}^{i}$ : solar system spatial (harmonic) coordinates. The proper time of a clock A located at the GRS coordinate position $\boldsymbol{x}_{\mathrm{A}}(t)$, and moving with the coordinate velocity $\boldsymbol{v}_{\mathrm{A}}=\mathrm{d} \boldsymbol{x}_{\mathrm{A}} / \mathrm{d} t$, is

$$
\begin{aligned}
\frac{\mathrm{d} \tau_{\mathrm{A}}}{\mathrm{d} t}= & 1-\frac{1}{c^{2}}\left[\frac{\boldsymbol{v}_{\mathrm{A}}^{2}}{2}+U_{\mathrm{E}}\left(\boldsymbol{x}_{\mathrm{A}}\right)\right. \\
& \left.+V\left(\mathcal{X}_{\mathrm{A}}\right)-V\left(\mathcal{X}_{\mathrm{E}}\right)-x_{\mathrm{A}}^{i} \partial_{i} V\left(\mathcal{X}_{\mathrm{E}}\right)+x_{\mathrm{A}}^{i} Q_{i}\right]
\end{aligned}
$$

Here, $U_{\mathrm{E}}$ denotes the Newtonian potential of the Earth at the position $\boldsymbol{x}_{\mathrm{A}}$ of the clock in the GRS frame, and $V$ is the sum of the Newtonian potentials of the other bodies (mainly the Sun and the Moon), either at the position $\mathcal{X}_{\mathrm{E}}$ of the Earth center of mass in barycentric coordinates, or at the clock location $\mathcal{X}_{\mathrm{A}}$. The three terms involving the

1 The relation between $t$ and the terrestrial time TT (realized by the International Atomic Time, TAI) is given by $\mathrm{d} t / \mathrm{dTT}=1+L_{\mathrm{g}}$ where $L_{\mathrm{g}}$ is a defining constant fixed in IAU resolution B1.9 (IAU 2000) as $L_{\mathrm{g}}=6.96929013410^{-10}$; this value was chosen to be close to the previous definition $L_{\mathrm{g}}=W_{0} / c^{2}$ (IAU 1991) where $W_{0}$ is the Earth potential (gravitational plus centrifugal) at the reference geoid close to the mean surface of the oceans. 
potential $V$ represent the tidal field of the other bodies at the position of the clock. [To a good approximation (introducing errors of much less than $10^{-18}$ ) one can write $V\left(\mathcal{X}_{\mathrm{A}}\right) \approx V\left(\mathcal{X}_{\mathrm{E}}+\boldsymbol{x}_{\mathrm{A}}\right)$. Then, as usual, the tidal field at the position $\boldsymbol{x}_{\mathrm{A}}$ can be approximated using a Taylor expansion by the standard expression $\frac{1}{2} x_{\mathrm{A}}^{i} x_{\mathrm{A}}^{j} \partial_{i j} V\left(\mathcal{X}_{\mathrm{E}}\right)$ again neglecting terms smaller than $10^{-18}$.] The terms due to the tides of the other bodies (i.e. involving $V$ ) are small for the ground station and the low orbit of ACES (of order $210^{-17}$ ) and are either negligible or easily evaluated if required (depending on the final clock accuracies reached). The last term in (2) is due to the non-geodesic acceleration of the center of mass of the Earth that is induced by the mass quadrupole of the Earth ${ }^{2}$, and which is numerically of the order of $310^{-11} \mathrm{~m} / \mathrm{s}^{2}$. This term is negligible in the case of the envisioned experiments: numerically $\left|Q_{i} x_{\mathrm{A}}^{i} / c^{2}\right|$ amounts to less that $10^{-20}$.

Thus, for application to ACES, and more generally to any experiment at a level of uncertainty greater than $510^{-17}$ on a satellite at similar altitude as ACES, we can keep only the first three terms in the relation (2) between the proper time $\tau_{\mathrm{A}}$ and the coordinate time $t$ :

$$
\frac{\mathrm{d} \tau_{\mathrm{A}}}{\mathrm{d} t}=1-\frac{1}{c^{2}}\left[\frac{\boldsymbol{v}_{\mathrm{A}}^{2}}{2}+U_{\mathrm{E}}\left(\boldsymbol{x}_{\mathrm{A}}\right)\right] .
$$

Note that at this level of uncertainty it is crucial to take into account in the relation (3) the non-sphericity (oblaticity) of the Earth Newtonian potential. In fact, it is even not sufficient to model the Earth potential with a $J_{2}$-term taking into account the quadrupolar deformation. Rather, the potential $U_{\mathrm{E}}\left(\boldsymbol{x}_{\mathrm{A}}\right)$ should be computed according to the detailed procedures of Wolf \& Petit (1995), and Petit \& Wolf (1997). For example, for a clock B fixed on the Earth surface, the relativistic correction term appearing in the coordinate/proper time relation (3) is given with the required precision by

$$
\frac{\boldsymbol{v}_{\mathrm{B}}^{2}}{2}+U_{\mathrm{E}}\left(\boldsymbol{x}_{\mathrm{B}}\right)=W_{0}-\int_{0}^{H_{\mathrm{B}}} g \mathrm{~d} H
$$

where $W_{0}$ is the Earth potential at the reference geoid $\left(W_{0}=62636856 \mathrm{~m}^{2} / \mathrm{s}^{2}\right)$, where $g$ denotes the (gravitational plus centrifugal) Earth acceleration, and where $H_{\mathrm{B}}$ is the geometric height of the clock above the reference geoid. Note that this procedure is limited by the uncertainty in the determination of $W_{0}$ which at present gives rise to an error of about 1 part in $10^{17}$ in $\mathrm{d} \tau_{\mathrm{B}} / \mathrm{d} t$. For uncertainties below that level, it is expected that, inversely, clock comparisons with highly accurate space clocks will yield the best estimate of the potential on the ground.

2 To a sufficient approximation we have

$Q_{i}=-\frac{1}{2 M_{\mathrm{E}}} I_{\mathrm{E}}^{j k} \partial_{i j k} V\left(\mathcal{X}_{\mathrm{E}}\right)$,

where $I_{\mathrm{E}}^{j k}$ is the Earth quadrupole moment (see e.g. Brumberg \& Kopejkin 1990 for details).

\section{Coordinate time transfer}

\subsection{One-way signal transmission}

Let A be the emitting station, with GRS position $\boldsymbol{x}_{\mathrm{A}}(t)$, and $\mathrm{B}$ the receiving station, with position $\boldsymbol{x}_{\mathrm{B}}(t)$. We denote by $t_{\mathrm{A}}$ the coordinate time at the instant of emission of a light signal, and by $t_{\mathrm{B}}$ the coordinate time at the instant of reception. We put $r_{\mathrm{A}}=\left|\boldsymbol{x}_{\mathrm{A}}\left(t_{\mathrm{A}}\right)\right|, r_{\mathrm{B}}=\left|\boldsymbol{x}_{\mathrm{B}}\left(t_{\mathrm{B}}\right)\right|$ and $R_{\mathrm{AB}}=\left|\boldsymbol{x}_{\mathrm{B}}\left(t_{\mathrm{B}}\right)-\boldsymbol{x}_{\mathrm{A}}\left(t_{\mathrm{A}}\right)\right|$, where || is the Euclidean norm associated with the metric $\delta_{i j}$. Up to the order $1 / c^{3}$ the coordinate time transfer $T_{\mathrm{AB}} \equiv t_{\mathrm{B}}-t_{\mathrm{A}}$ is given by

$T_{\mathrm{AB}}=\frac{R_{\mathrm{AB}}}{c}+\frac{2 G M_{\mathrm{E}}}{c^{3}} \ln \left(\frac{r_{\mathrm{A}}+r_{\mathrm{B}}+R_{\mathrm{AB}}}{r_{\mathrm{A}}+r_{\mathrm{B}}-R_{\mathrm{AB}}}\right)$,

where the logarithmic term represents the Shapiro time delay $^{3}$ (Shapiro 1964). See the Appendix for several derivations of the Shapiro time delay and for an alternative expression given by (A.40). In the case of zenithal geometry, i.e. propagation of the signal along the local vertical (for which $\left|\boldsymbol{x}_{\mathrm{B}}-\boldsymbol{x}_{\mathrm{A}}\right|=\left|r_{\mathrm{B}}-r_{\mathrm{A}}\right|$ ), between the orbit of ACES at $400 \mathrm{~km}$ (assumed in all numerical examples below) and the ground, the Shapiro time delay is 2 ps. In the case of zero elevation, it is 11 ps.

In a real experiment, the position of the receptor $B$ may be recorded at the time of emission $t_{\mathrm{A}}$ rather than at the time of reception $t_{\mathrm{B}}$, i.e. we may have more direct access to $\boldsymbol{x}_{\mathrm{B}}\left(t_{\mathrm{A}}\right)$ rather than $\boldsymbol{x}_{\mathrm{B}}\left(t_{\mathrm{B}}\right)$, and the formula (5) gets modified by some Sagnac correction terms consistently to the order $1 / c^{3}$. In this case the formula becomes

$$
\begin{aligned}
T_{\mathrm{AB}}= & \frac{D_{\mathrm{AB}}}{c}+\frac{\boldsymbol{D}_{\mathrm{AB}} \cdot \boldsymbol{v}_{\mathrm{B}}\left(t_{\mathrm{A}}\right)}{c^{2}} \\
& +\frac{D_{\mathrm{AB}}}{2 c^{3}}\left(\boldsymbol{v}_{\mathrm{B}}^{2}+\frac{\left(\boldsymbol{D}_{\mathrm{AB}} \cdot \boldsymbol{v}_{\mathrm{B}}\right)^{2}}{D_{\mathrm{AB}}^{2}}+\boldsymbol{D}_{\mathrm{AB}} \cdot \boldsymbol{a}_{\mathrm{B}}\right) \\
& +\frac{2 G M_{\mathrm{E}}}{c^{3}} \ln \left(\frac{r_{\mathrm{A}}+r_{\mathrm{B}}+D_{\mathrm{AB}}}{r_{\mathrm{A}}+r_{\mathrm{B}}-D_{\mathrm{AB}}}\right)
\end{aligned}
$$

where $\boldsymbol{D}_{\mathrm{AB}}=\boldsymbol{x}_{\mathrm{B}}\left(t_{\mathrm{A}}\right)-\boldsymbol{x}_{\mathrm{A}}\left(t_{\mathrm{A}}\right)$ is the "instantaneous" coordinate distance between $\mathrm{A}$ and $\mathrm{B}$ at the instant of emission at $\mathrm{A}$ (we have $D_{\mathrm{AB}}=\left|\boldsymbol{D}_{\mathrm{AB}}\right|$ ), where $\boldsymbol{v}_{\mathrm{B}}\left(t_{\mathrm{A}}\right)$ denotes the coordinate velocity of the station $\mathrm{B}$ at that instant, and where $\boldsymbol{a}_{\mathrm{B}}$ is the acceleration of B (in all the small correction terms of order $1 / c^{3}$ one can use, independent of the required order, the quantities at the instants $t_{\mathrm{A}}$ or $\left.t_{\mathrm{B}}\right)$. The second term in (6) represents the Sagnac term of order $1 / c^{2}$ and can amount to $200 \mathrm{~ns}$ at low elevation; the third term or Sagnac of order $1 / c^{3}$ is about 5 ps at low elevation (to be compared with the Shapiro term, which is $11 \mathrm{ps}$ ).

\subsection{Two-way time transfer}

One signal is emitted from the satellite $\mathrm{A}$ at instant $t_{\mathrm{A}}$ and received by the ground station $\mathrm{B}$ at instant $t_{\mathrm{B}}$. A second

\footnotetext{
${ }^{3}$ In the case of a general metric theory of gravity, the factor two in front of the Shapiro time delay should be replaced by $1+\gamma$ where $\gamma$ is the standard PPN parameter.
} 


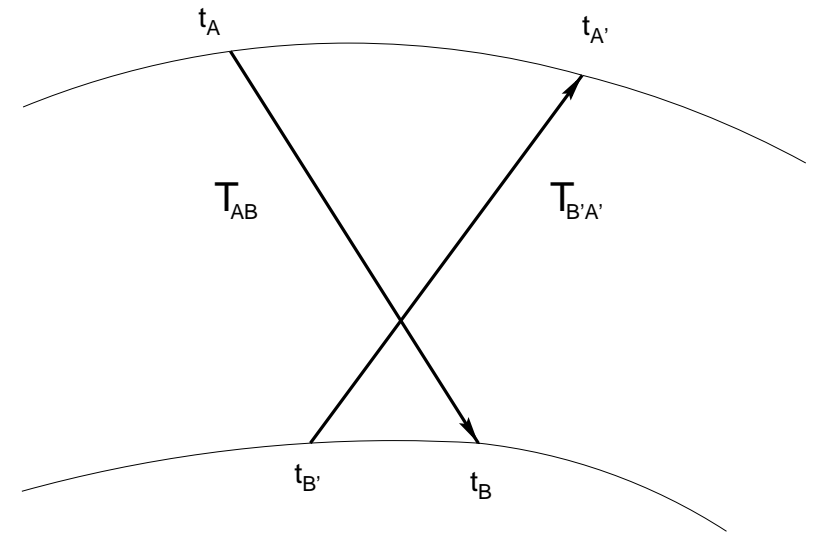

Fig. 1. Two-way time transfer in the non-rotating frame

signal (prime) is emitted by the ground $\mathrm{B}$ at instant $t_{\mathrm{B}^{\prime}}$ and received on $\mathrm{A}$ at $t_{\mathrm{A}^{\prime}}$ (see Fig. 1). The two transmission times are

$$
T_{\mathrm{AB}}=t_{\mathrm{B}}-t_{\mathrm{A}}
$$

$T_{\mathrm{B}^{\prime} \mathrm{A}^{\prime}}=t_{\mathrm{A}^{\prime}}-t_{\mathrm{B}^{\prime}}$.

Furthermore, we denote the intervals of time between emission and reception on board the satellite $\mathrm{A}$ and at the station B by

$t_{\mathrm{AA}^{\prime}}=t_{\mathrm{A}^{\prime}}-t_{A}$

$t_{\mathrm{B}^{\prime} \mathrm{B}}=t_{\mathrm{B}}-t_{\mathrm{B}^{\prime}}$

From these definitions we deduce the quantity $\Delta t$ which is required for synchronization, namely

$\Delta t=t_{\mathrm{A}}-t_{\mathrm{B}^{\prime}}=\frac{1}{2}\left(t_{\mathrm{B}^{\prime} \mathrm{B}}-t_{\mathrm{AA}^{\prime}}+T_{\mathrm{B}^{\prime} \mathrm{A}^{\prime}}-T_{\mathrm{AB}}\right)$.

Notice that $\Delta t$ is known because it is expressed in terms of the two transmission times $T_{\mathrm{AB}}$ and $T_{\mathrm{B}^{\prime} \mathrm{A}^{\prime}}$ which are computed from the theoretical formulas (5) or (6) valid for the one-way time transfer, and in terms of the time intervals $t_{\mathrm{AA}^{\prime}}$ and $t_{\mathrm{B}^{\prime} \mathrm{B}}$ which are measured on the satellite and on the ground respectively ${ }^{4}$.

\section{Frequency transfer}

\subsection{One-way transfer}

The frequency transfer between two clocks requires the determination of the ratio $f_{\mathrm{A}} / f_{\mathrm{B}}$ between the proper frequencies $f_{\mathrm{A}}$ and $f_{\mathrm{B}}$ delivered by the clocks on the satellite (A) and on the ground (B). In practice this is achieved using a transmission of photons from $\mathrm{A}$ to $\mathrm{B}$ and the formula

$\frac{f_{\mathrm{A}}}{f_{\mathrm{B}}}=\left(\frac{f_{\mathrm{A}}}{\nu_{\mathrm{A}}}\right)\left(\frac{\nu_{\mathrm{A}}}{\nu_{\mathrm{B}}}\right)\left(\frac{\nu_{\mathrm{B}}}{f_{\mathrm{B}}}\right)$,

where $\nu_{\mathrm{A}}$ is the proper frequency of the photon as measured on $\mathrm{A}$ (instant of emission $t_{\mathrm{A}}$ ), and $\nu_{\mathrm{B}}$ the proper

\footnotetext{
${ }^{4}$ In the case of the time transfer by laser light T2L2 to be operated on ACES we have $t_{\mathrm{AA}^{\prime}}=0$ since the signal is reflected instantaneously.
}

frequency of the same photon on $\mathrm{B}$ at $t_{\mathrm{B}}$. The first bracket in (12) is measured on A, the second bracket is given by the theoretical formula (13) below, and the third one is measured on B. In the one-way transfer of photons, as derived in the Appendix, we have

$$
\frac{\nu_{\mathrm{A}}}{\nu_{\mathrm{B}}}=\frac{1-\frac{1}{c^{2}}\left[U_{\mathrm{E}}\left(\boldsymbol{r}_{\mathrm{B}}\right)+\frac{\boldsymbol{v}_{\mathrm{B}}^{2}}{2}\right]}{1-\frac{1}{c^{2}}\left[U_{\mathrm{E}}\left(\boldsymbol{r}_{\mathrm{A}}\right)+\frac{\boldsymbol{v}_{\mathrm{A}}^{2}}{2}\right]} \frac{q_{\mathrm{A}}}{q_{\mathrm{B}}} .
$$

For convenience in the notation, we henceforth denote the radial vectors (in GRS coordinates) by $\boldsymbol{r}_{\mathrm{A}}=\boldsymbol{x}_{\mathrm{A}}\left(t_{\mathrm{A}}\right)$ and $\boldsymbol{r}_{\mathrm{B}}=\boldsymbol{x}_{\mathrm{B}}\left(t_{\mathrm{B}}\right)$; and, as before, we have $r_{\mathrm{A}}=\left|\boldsymbol{r}_{\mathrm{A}}\right|$ and $r_{B}=$ $\left|\boldsymbol{r}_{B}\right|$, as well as the coordinate velocities $\boldsymbol{v}_{\mathrm{A}}=\boldsymbol{v}_{\mathrm{A}}\left(t_{\mathrm{A}}\right)$ and $\boldsymbol{v}_{\mathrm{B}}=\boldsymbol{v}_{\mathrm{B}}\left(t_{\mathrm{B}}\right)$. To the required order $1 / c^{3}$, the last factor in (13) is obtained from

$$
\begin{aligned}
q_{\mathrm{A}}=1 & -\frac{\boldsymbol{N}_{\mathrm{AB}} \cdot \boldsymbol{v}_{\mathrm{A}}}{c} \\
& -\frac{4 G M_{\mathrm{E}}}{c^{3}} \frac{\left(r_{\mathrm{A}}+r_{\mathrm{B}}\right) \boldsymbol{N}_{\mathrm{AB}} \cdot \boldsymbol{v}_{\mathrm{A}}+R_{\mathrm{AB}} \frac{\boldsymbol{r}_{\mathrm{A}} \cdot \boldsymbol{v}_{\mathrm{A}}}{r_{\mathrm{A}}}}{\left(r_{\mathrm{A}}+r_{\mathrm{B}}\right)^{2}-R_{\mathrm{AB}}^{2}} \\
q_{\mathrm{B}}=1 & -\frac{\boldsymbol{N}_{\mathrm{AB}} \cdot \boldsymbol{v}_{\mathrm{B}}}{c} \\
& -\frac{4 G M_{\mathrm{E}}}{c^{3}} \frac{\left(r_{\mathrm{A}}+r_{\mathrm{B}}\right) \boldsymbol{N}_{\mathrm{AB}} \cdot \boldsymbol{v}_{\mathrm{B}}-R_{\mathrm{AB}} \frac{\boldsymbol{r}_{\mathrm{B}} \cdot \boldsymbol{v}_{\mathrm{B}}}{r_{\mathrm{B}}}}{\left(r_{\mathrm{A}}+r_{\mathrm{B}}\right)^{2}-R_{\mathrm{AB}}^{2}}
\end{aligned}
$$

with $\boldsymbol{R}_{\mathrm{AB}}=\boldsymbol{r}_{\mathrm{B}}-\boldsymbol{r}_{\mathrm{A}}, R_{\mathrm{AB}}=\left|\boldsymbol{R}_{\mathrm{AB}}\right|$ and $\boldsymbol{N}_{\mathrm{AB}}=$ $\boldsymbol{R}_{\mathrm{AB}} / R_{\mathrm{AB}}$. See the Appendix for the derivation of these formulas. Note that the result (14)-(15) has been obtained in the Appendix assuming that the field of the Earth is spherically symmetric. Indeed, the $J_{2}$-terms in the factor $q_{\mathrm{A}} / q_{\mathrm{B}}$ do not exceed $410^{-17}$.

In the case of ACES, the various contributions in the one-way frequency transfer (13)-(15) are numerically as follows. First-order Doppler effect: for the satellite $\left|\boldsymbol{N}_{\mathrm{AB}} \cdot \boldsymbol{v}_{\mathrm{A}} / c\right| \leq 2.610^{-5}$; for the ground $\left|\boldsymbol{N}_{\mathrm{AB}} \cdot \boldsymbol{v}_{\mathrm{B}} / c\right| \leq$ $1.610^{-6}$. Second-order Doppler effect: $\boldsymbol{v}_{\mathrm{A}}^{2} /\left(2 c^{2}\right) \leq$ $3.410^{-10}$ for the satellite; $\boldsymbol{v}_{\mathrm{B}}^{2} /\left(2 c^{2}\right) \leq 1.310^{-12}$ for the ground. Gravitational red-shift (Einstein) effect: $U_{\mathrm{A}} / c^{2} \equiv$ $U_{\mathrm{E}}\left(\boldsymbol{r}_{\mathrm{A}}\right) / c^{2}=6.510^{-10} ; U_{\mathrm{B}} / c^{2}=6.910^{-10}$. The terms of order $1 / c^{3}$ are less than $3.610^{-14}$ for the satellite and $2.210^{-15}$ for the ground.

\subsection{Two-way frequency transfer}

A "tracking" signal is sent from the ground station B at instant $t_{\mathrm{B}^{\prime}}$, received on the satellite $\mathrm{A}$ at instant $t_{\mathrm{A}}$ and instantaneously re-emitted by a satellite transponder toward $\mathrm{B}$ where it is received at instant $t_{\mathrm{B}}$. The down-link "clock" signal is emitted simultaneously with the tracking signal at the transponding instant $t_{\mathrm{A}}$, and received at $t_{\mathrm{B}}$ (see Fig. 2) (Vessot et al. 1980). In the two-way frequency transfer the ratio $\nu_{\mathrm{A}} / \nu_{\mathrm{B}}$ in (12), needed for frequency comparison, is given by

$\frac{\nu_{\mathrm{B}}}{\nu_{\mathrm{A}}}=\frac{1}{2}\left(\frac{\nu_{\mathrm{B}}}{\nu_{\mathrm{B}^{\prime}}}\right)+\Delta_{\mathrm{AB}}+\frac{1}{2}$.

The ratio $\left(\nu_{\mathrm{B}} / \nu_{\mathrm{B}^{\prime}}\right)=\left(\nu_{\mathrm{B}} / \nu_{\mathrm{B}^{\prime}}\right)_{\text {station }}$ is measured at the ground station $\mathrm{B}$, while $\Delta_{\mathrm{AB}}$ is computed by means of the 


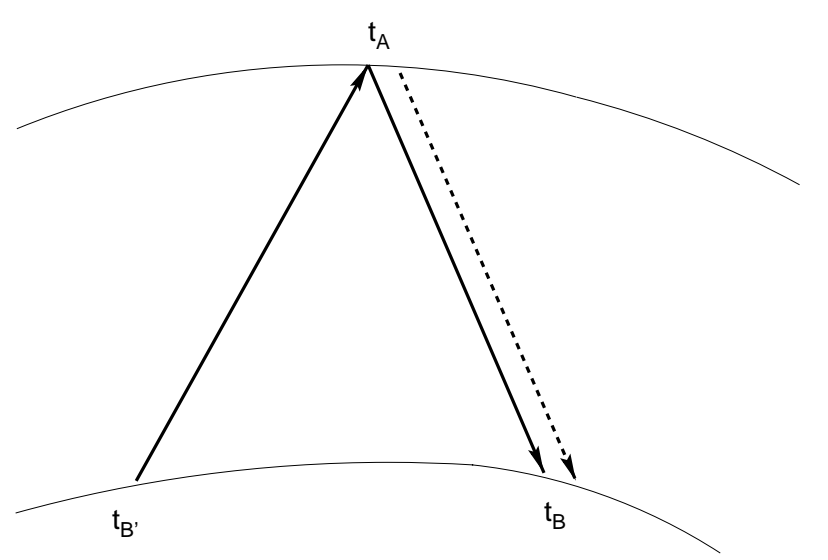

Fig. 2. Two-way frequency transfer in the non-rotating frame

theoretical formula (derived in the Appendix)

$$
\begin{aligned}
\Delta_{\mathrm{AB}}= & \frac{1}{c^{2}}\left[U_{\mathrm{AB}}-\frac{1}{2} \boldsymbol{v}_{\mathrm{AB}}^{2}-\boldsymbol{R}_{\mathrm{AB}} \cdot \boldsymbol{a}_{\mathrm{B}}\right]\left(1+\frac{\boldsymbol{N}_{\mathrm{AB}} \cdot \boldsymbol{v}_{\mathrm{AB}}}{c}\right) \\
& +\frac{R_{\mathrm{AB}}}{c^{3}}\left(-\boldsymbol{v}_{\mathrm{A}} \cdot \boldsymbol{a}_{\mathrm{B}}+\boldsymbol{R}_{\mathrm{AB}} \cdot \boldsymbol{b}_{\mathrm{B}}+2 \boldsymbol{v}_{\mathrm{B}} \cdot \boldsymbol{a}_{\mathrm{B}}-\boldsymbol{v}_{\mathrm{B}} \cdot \nabla U_{\mathrm{B}}\right) .
\end{aligned}
$$

The difference of potentials between ground and satellite reads $U_{\mathrm{AB}}=U_{\mathrm{B}}-U_{\mathrm{A}}$ where $U_{\mathrm{A}} \equiv U_{\mathrm{E}}\left[\boldsymbol{r}_{\mathrm{A}}\left(t_{\mathrm{A}}\right)\right]$ and $U_{\mathrm{B}} \equiv$ $U_{\mathrm{E}}\left[\boldsymbol{r}_{\mathrm{B}}\left(t_{\mathrm{B}}\right)\right]$ (note that $U_{\mathrm{AB}}>0$ ); the gradient is $\nabla U_{\mathrm{B}}=$ $\partial U_{\mathrm{E}}\left(\boldsymbol{r}_{\mathrm{B}}\right) / \partial \boldsymbol{r}_{\mathrm{B}}$; the relative velocity is $\boldsymbol{v}_{\mathrm{AB}}=\boldsymbol{v}_{\mathrm{A}}\left(t_{\mathrm{A}}\right)-$ $\boldsymbol{v}_{\mathrm{B}}\left(t_{\mathrm{B}}\right)$; the acceleration of the ground is $\boldsymbol{a}_{\mathrm{B}}=\boldsymbol{a}_{\mathrm{B}}\left(t_{\mathrm{B}}\right)$; the derivative of acceleration is $\boldsymbol{b}_{\mathrm{B}}=\mathrm{d} \boldsymbol{a}_{\mathrm{B}} / \mathrm{d} t$.

In the case of ACES we find the following numerical contributions. For the three dominant terms appearing at the order $1 / c^{2}$,

$$
\begin{aligned}
& \Delta_{\text {Einstein }}=\frac{U_{\mathrm{AB}}}{c^{2}}=4.610^{-11} ; \\
& \Delta_{2 \mathrm{~d} \text {-order Doppler }}=\left|-\frac{1}{2 c^{2}} \boldsymbol{v}_{\mathrm{AB}}^{2}\right| \leq 3.310^{-10} ; \\
& \Delta_{\text {acceleration }}=\left|-\frac{1}{c^{2}} \boldsymbol{R}_{\mathrm{AB}} \cdot \boldsymbol{a}_{\mathrm{B}}\right| \leq 710^{-13} .
\end{aligned}
$$

In the third term, or "acceleration" term, we have assumed that the station $\mathrm{B}$ is located at the equator, and we have used the upper bound $\left(R_{\mathrm{AB}}\right)_{\max } \omega_{\mathrm{E}}^{2} r_{\mathrm{B}} / c^{2}$, where $\omega_{\mathrm{E}}$ is the Earth's angular velocity of rotation. Now, according to (17), in order to reach the $1 / c^{3}$ precision, the three previous terms are to be corrected by a factor that looks exactly like a first-order Doppler effect. Numerically, we have

$$
\left|\frac{\boldsymbol{N}_{\mathrm{AB}} \cdot \boldsymbol{v}_{\mathrm{AB}}}{c}\right| \leq 2.710^{-5},
$$

so this first-order Doppler factor induces a correction of the frequency shift at the minimal level $8.210^{-15}$, which is measurable by ACES. Finally the four last terms in (17) are purely of order $1 / c^{3}$. They amount respectively to the maximal values $\leq 210^{-17}, 3.510^{-19}$, and much less for the last two terms. These last four terms are in general negligible for ACES. The terms which are neglected in the formula (17), which are at least $\mathcal{O}\left(1 / c^{4}\right)$, are numerically of the order of $10^{-20}$ or less, too small to be detected by ACES.

In summary, the formula (17) and its derivation in the Appendix constitute the main results of this paper. To the dominant order $1 / c^{2}$ the expression was first derived by Vessot et al. (1980) who used it in their GP-A experiment. To the order $1 / c^{3}$ the formula (17) appears in a recent paper by Ashby (1998) but without detailed derivation. The final expression (17) is relatively simple, as compared, for instance, to the corresponding expression valid for the one-way frequency transfer [see (13)-(15)]. We find indeed that many of the scalar products between $\boldsymbol{N}_{\mathrm{AB}}$ and some velocities, though present in the intermediate steps of the calculation, drop out from the final result. The exception is for the factor in (17) which is made of the combination $1+\boldsymbol{N}_{\mathrm{AB}} \cdot \boldsymbol{v}_{\mathrm{AB}} / c$ and which can be nicely interpreted as a modification, at the level $1 / c^{3}$, of the dominant term of order $1 / c^{2}$ by a first-order Doppler effect. The set of equations for time and frequency transfers given in this paper should be sufficient for the analysis of the planned clock experiments in Earth orbit, at the level $510^{-17}$ in fractional frequency.

\section{Appendix A: Theory}

This Appendix presents several equivalent derivations of the formulas for the time and frequency transfers in the ACES experiment. Some of the basic material needed in these derivations is not new and can be found in standard textbooks such as Misner et al. (1973), and Will (1981). On the other hand, the problem of the propagation of light in a gravitational field has been solved in a general way at the linearized approximation: see Kopeikin \& Schäfer (1999) for a complete investigation and an entry to the literature. Here we use the explicit solution of the photon motion, the optical distance function for stationary spacetimes (Buchdahl 1970,1979), and the differentiation of the well-known Shapiro (1964) formula.

We shall first consider the transfer of coordinate geocentric time $t$; for this purpose it is sufficient to approximate the gravitational field of the Earth as spherically symmetric (monopolar), and to neglect tidal terms, hence $U_{\mathrm{E}}=G M / r$. However, as we have seen in Sect. 2, higher spherical harmonics in the Earth potential are needed in the relation between coordinate and proper time. We denote the mass of the Earth by $M=M_{\mathrm{E}}$, the Cartesian geocentric coordinates by $(t, \boldsymbol{r})$, with $t=$ TCG and $\boldsymbol{r}=\boldsymbol{x}$ agreeing to this approximation with the geocentric spatial coordinates $(r=|\boldsymbol{r}|)$. Using spherical coordinates $\{t, r, \theta, \varphi\}$ associated in the standard way to the geocentric coordinates $(0 \leq \theta \leq \pi, 0 \leq \varphi \leq 2 \pi)$, we can write the $1 \mathrm{PN}$ metric as

$$
\mathrm{d} s^{2}=-f(r) c^{2} \mathrm{~d} t^{2}+g(r)\left[\mathrm{d} r^{2}+r^{2} \mathrm{~d} \theta^{2}+r^{2} \sin ^{2} \theta \mathrm{d} \varphi^{2}\right]
$$

where to this order

$f(r)=1-\frac{2 U}{c^{2}}=1-\frac{2 G M}{r c^{2}}$, 
$g(r)=1+\frac{2 U}{c^{2}}=1+\frac{2 G M}{r c^{2}}$,

As said above, the monopolar approximation to the Earth potential is sufficient for our purpose in most of this Appendix.

Photon equations of motion. In the geometric optics approximation, where the photon's wavelength is much smaller than the typical size of the electromagnetic wave packet, as well as of the space-time radius of curvature, the photon's wave vector is null, $k_{\mu} k^{\mu}=0$, and is hypersurface-orthogonal, $k_{\mu}=\partial_{\mu} S$. The differential equation for the trajectory of the ray is $k^{\mu}=\mathrm{d} x^{\mu} / \mathrm{d} p=$ $g^{\mu \nu}(x) \partial_{\nu} S(x)$. We have $0=\nabla_{\mu}\left(k_{\nu} k^{\nu}\right)=2 k^{\nu} \nabla_{\mu} k_{\nu}=$ $2 k^{\nu} \nabla_{\nu} k_{\mu}$, where we have used the fact that $\nabla_{\mu} k_{\nu}$ is symmetric as a consequence of $k_{\mu}=\partial_{\mu} S$, so we find that $k^{\nu} \nabla_{\nu} k_{\mu}=0$, which is the geodesic equation, a more useful form of which reads

$\frac{\mathrm{d} k_{\mu}}{\mathrm{d} p}=\frac{1}{2} k^{\rho} k^{\sigma} \partial_{\mu} g_{\rho \sigma}$.

Here, $p$ denotes an affine parameter along the trajectory, and the photon's null wave vector $k^{\mu}$ is such that

$k^{\mu}=\frac{\mathrm{d} x^{\mu}}{\mathrm{d} p} ; \quad k_{\mu}=g_{\mu \nu} k^{\nu} ; \quad 0=k_{\mu} k^{\mu}$,

( $k^{\mu}$ is future directed, $k^{0}=c k^{t}>0$ ).

Although the metric (A.1)-(A.3) is sphericallysymmetric, it is convenient to suppose that the motion of the photon takes place in an arbitrary plane, not necessarily the equatorial plane $\theta=\pi / 2$. For instance, we can consider that $\theta=0$ represents the Earth's rotation axis, and that the emitted signal comes from a satellite moving on any orbit with inclination angle $i$ with respect to the equator. Since the metric is stationary and axi-symmetric, we find immediately the first integrals $k_{t}=-\hat{E}$ and $k_{\varphi}=\hat{L}$, where $\hat{E}$ and $\hat{L}$ denote two constants along the trajectory (with $\hat{E}>0$ to ensure $k^{0}>0$ ). Relating $k_{t}$ and $k_{\varphi}$ to the contravariant components of the wave vector, $k^{t}=\mathrm{d} t / \mathrm{d} p$ and $k^{\varphi}=\mathrm{d} \varphi / \mathrm{d} p$, yields the two integrals of motion

$$
\begin{aligned}
& \hat{E}=f(r) c^{2} \frac{\mathrm{d} t}{\mathrm{~d} p}, \\
& \hat{L}=g(r) r^{2} \sin ^{2} \theta \frac{\mathrm{d} \varphi}{\mathrm{d} p} .
\end{aligned}
$$

Next, the equation corresponding to the $\theta$-coordinate reads $\mathrm{d} k_{\theta} / \mathrm{d} p=g(r) r^{2} \sin \theta \cos \theta\left(k^{\varphi}\right)^{2}$. Inserting in this equation $k^{\varphi}=\mathrm{d} \varphi / \mathrm{d} p$ as deduced from (A.7), and noting that $g(r) r^{2}=g_{\theta \theta}$, we obtain $\mathrm{d}\left(k_{\theta}^{2}\right) / \mathrm{d} p=$ $2 \hat{L}^{2} \cos \theta / \sin ^{3} \theta \mathrm{d} \theta / \mathrm{d} p$. This equation shows that $k_{\theta}$ is a function of the $\theta$-coordinate only, and we get $k_{\theta}^{2}=$ $\hat{b}^{2}-\hat{L}^{2} / \sin ^{2} \theta$ where $\hat{b}$ is a new constant of the motion. Hence,

$$
r^{4} g^{2}(r)\left(\frac{\mathrm{d} \theta}{\mathrm{d} p}\right)^{2}=\hat{b}^{2}-\frac{\hat{L}^{2}}{\sin ^{2} \theta} .
$$

Finally, since $g^{\mu \nu} k_{\mu} k_{\nu}=0$ along a light ray, we get from the previous integrals of motion,

$g^{2}(r)\left(\frac{\mathrm{d} r}{\mathrm{~d} p}\right)^{2}=\frac{g(r)}{f(r)} \frac{\hat{E}^{2}}{c^{2}}-\frac{\hat{b}^{2}}{r^{2}}$

It can be checked that the equation concerning the $r$ coordinate, i.e. $\mathrm{d} k_{r} / \mathrm{d} p=1 / 2 k^{\rho} k^{\sigma} \partial_{r} g_{\rho \sigma}$, is now automatically satisfied. Thus, the photon motion depends on the constants $\hat{E}, \hat{b}$ and $\hat{L}$; however, by eliminating the affine parameter $p$ in favor of the coordinate time $t$ we can parametrize the motion by only two constants, say $b=\hat{b} c / \hat{E}$ and $L=\hat{L} c / \hat{E}$. Finally, the solution reads

$$
\begin{aligned}
\frac{\mathrm{d} r}{c \mathrm{~d} t} & =\varepsilon_{r} \frac{f}{g} \sqrt{\frac{g}{f}-\frac{b^{2}}{r^{2}}}, \\
r^{2} \frac{\mathrm{d} \theta}{c \mathrm{~d} t} & =\varepsilon_{\theta} \frac{f}{g} \sqrt{b^{2}-\frac{L^{2}}{\sin ^{2} \theta}}, \\
r^{2} \frac{\mathrm{d} \varphi}{c \mathrm{~d} t} & =\frac{f}{g} \frac{L}{\sin ^{2} \theta} .
\end{aligned}
$$

We have introduced the signs $\varepsilon_{r}$ and $\varepsilon_{\theta}$ of $\mathrm{d} r / \mathrm{d} t$ and $\mathrm{d} \theta / \mathrm{d} t$. Because the metric (A.1) is spherically-symmetric, one could rotate the coordinates so that the plane of the motion is simply the equatorial plane $\theta=\pi / 2$. This would correspond to $b_{\text {equatorial }}=L$, and in that case $\varphi$ would simply be the polar angle within the orbital plane. In our more general situation, it is not difficult to find the equation of the orbital plane. We introduce the angle $\alpha$ defined by

$$
\cos \alpha=\frac{b \cos \theta}{\sqrt{b^{2}-L^{2}}} ; \quad \sin \alpha=\varepsilon_{\theta} \sqrt{\frac{b^{2} \sin ^{2} \theta-L^{2}}{b^{2}-L^{2}}} .
$$

In terms of $\alpha$ we can integrate the equation for the azimuthal angle $\varphi$ in the form

$\tan \left(\varphi-\varphi_{1}\right)=\frac{b}{L} \tan \alpha$

where $\varphi_{1}$ is an arbitrary constant. Clearly this is the equation of the orbital plane. Its line of node is defined by the direction $\varphi=\varphi_{1}-\pi / 2$ in the equatorial plane, and the inclination angle $i$ of the orbit is such that $\sin i=\sqrt{1-L^{2} / b^{2}}$. The angle $\alpha$ is the polar angle in the orbital plane, oriented in the sense of the motion (we have $\mathrm{d} \alpha^{2}=\mathrm{d} \theta^{2}+\sin ^{2} \theta \mathrm{d} \varphi^{2}$ ). Introducing the Euclidean orthonormal basis $\boldsymbol{e}_{r}, \boldsymbol{e}_{\theta}, \boldsymbol{e}_{\varphi}$ associated with the spherical coordinates $\{r, \theta, \varphi\}$, the position and velocity of the photon read

$$
\begin{aligned}
\boldsymbol{r} & =r \boldsymbol{e}_{r}, \\
\frac{\mathrm{d} \boldsymbol{r}}{c \mathrm{~d} t} & \equiv \boldsymbol{n} \sqrt{\frac{f(r)}{g(r)}}=\frac{\mathrm{d} r}{c \mathrm{~d} t} \boldsymbol{e}_{r}+r \frac{\mathrm{d} \alpha}{c \mathrm{~d} t} \boldsymbol{e}_{\alpha},
\end{aligned}
$$

where $\boldsymbol{e}_{\alpha}$, namely the unit vector in the direction of increasing $\alpha$ within the orbital plane, is given by

$\boldsymbol{e}_{\alpha}=\varepsilon_{\theta} \boldsymbol{e}_{\theta} \sqrt{1-\frac{L^{2}}{b^{2} \sin ^{2} \theta}}+\boldsymbol{e}_{\varphi} \frac{L}{b \sin \theta}$. 
In (A.16) we have introduced the unit tangent vector $\boldsymbol{n}$ along the photon's path, which is directly related to the contravariant components of the wave vector: $n^{i}=k^{i} /|\boldsymbol{k}|$, where $|\boldsymbol{k}|$ is the Euclidean norm, so that $\boldsymbol{n}^{2}=\delta_{i j} n^{i} n^{j}=1$. From the facts that $k^{\mu}$ is a null vector and that the line element (A.1) is diagonal and spatially conformally flat, we can check that $|\boldsymbol{k}|=\sqrt{f / g} k^{0}$, from which we deduce that the covariant components $k_{i}$ are also proportional to $n^{i}$, viz

$$
\frac{k_{i}}{k_{0}}=-\sqrt{\frac{g}{f}} n^{i}=-\frac{g}{f} \frac{k^{i}}{k^{0}}
$$

From its definition (A.16), and from the solution of the motion, we find that the tangent vector $\boldsymbol{n}$ is given by

$\boldsymbol{n}=\varepsilon_{r} \sqrt{1-\frac{f}{g} \frac{b^{2}}{r^{2}}} \boldsymbol{e}_{r}+\frac{b}{r} \sqrt{\frac{f}{g}} \boldsymbol{e}_{\alpha}$.

Next, the equations of motion for $r(t)$ and $\alpha(t)$ in the orbital plane are

$$
\begin{aligned}
\frac{\mathrm{d} r}{c \mathrm{~d} t} & =\varepsilon_{r} \frac{f}{g} \sqrt{\frac{g}{f}-\frac{b^{2}}{r^{2}}}, \\
r^{2} \frac{\mathrm{d} \alpha}{c \mathrm{~d} t} & =b \frac{f}{g} .
\end{aligned}
$$

The differential equation for the trajectory is

$$
\frac{\mathrm{d} r}{\mathrm{~d} \alpha}=\varepsilon_{r} r \sqrt{\frac{g}{f} \frac{r^{2}}{b^{2}}-1} .
$$

Photon trajectory. The trajectory at the relativistic order $1 / c^{2}$ (and even at order $1 / c^{3}$ ), which is the solution of (A.22), is an hyperbola whose focus is the center of the Earth, with impact parameter $b$ and total deviation angle $4 G M / c^{2} b$. The equation of the path reads

$\cos \left(\alpha-\alpha_{0}\right)=\frac{b}{r}-\frac{2 G M}{c^{2} b}$,

where the angle $\alpha_{0}$ represents the direction of the periastron, at which the distance of closest approach is $r_{0}$, which, to order $1 / c^{2}$, is given by

$r_{0}=b-\frac{2 G M}{c^{2}}$

The tangent vector along the trajectory can be expressed in terms of the vectors $\boldsymbol{e}_{\alpha_{0}}$ and $\boldsymbol{e}_{r_{0}}$, corresponding to the position $\left(r_{0}, \alpha_{0}\right)$ of the periastron:

$\boldsymbol{n}=\boldsymbol{e}_{\alpha_{0}}-\frac{2 G M}{c^{2} r_{0}} \sin \left(\alpha-\alpha_{0}\right) \boldsymbol{e}_{r_{0}}$.

As for the radial Eq. (A.20) it reads, after being expressed in terms of $r_{0}$ rather than $b$,

$c \mathrm{~d} t=\varepsilon_{r} \frac{r \mathrm{~d} r}{\sqrt{r^{2}-r_{0}^{2}}}\left[1+\frac{2 G M}{c^{2} r}\left(1+\frac{r_{0}}{r+r_{0}}\right)\right]$.
This is readily integrated as

$$
\begin{aligned}
c\left|t-t_{0}\right| & =\sqrt{r^{2}-r_{0}^{2}} \\
& +\frac{2 G M}{c^{2}}\left[\sqrt{\frac{r-r_{0}}{r+r_{0}}}+\ln \left(\frac{r+\sqrt{r^{2}-r_{0}^{2}}}{r_{0}}\right)\right]
\end{aligned}
$$

where $t_{0}$ denotes the instant of passage at the periastron. On the other hand, the trajectory is obtained as

$\left|\alpha-\alpha_{0}\right|=\arctan \left(\frac{\sqrt{r^{2}-r_{0}^{2}}}{r_{0}}\right)+\frac{2 G M}{c^{2} r_{0}} \sqrt{\frac{r-r_{0}}{r+r_{0}}}$,

where $\alpha_{0}$ is the angle at the periastron.

Coordinate time transfer. We consider from now on a transfer from an emission point A along the trajectory to some reception point $\mathrm{B}$. We introduce the Euclidean vectorial distance between the two points, defined as the difference of the coordinate positions of the points in the GRS coordinate system:

$\boldsymbol{R}_{\mathrm{AB}}=\boldsymbol{r}_{\mathrm{B}}-\boldsymbol{r}_{\mathrm{A}} ; R_{\mathrm{AB}}=\left|\boldsymbol{R}_{\mathrm{AB}}\right| ; \boldsymbol{N}_{\mathrm{AB}}=\boldsymbol{R}_{\mathrm{AB}} / R_{\mathrm{AB}}$.

The position of the emitting point $\mathrm{A}$ is taken at the time of emission $t_{\mathrm{A}}, \boldsymbol{r}_{\mathrm{A}}=\boldsymbol{r}_{\mathrm{A}}\left(t_{\mathrm{A}}\right)$, and similarly $\boldsymbol{r}_{\mathrm{B}}=\boldsymbol{r}_{\mathrm{B}}\left(t_{\mathrm{B}}\right)$. Notice that the norm $R_{\mathrm{AB}}$ is simply the Euclidean norm; thus, $R_{\mathrm{AB}}$ represents the "straight line" distance between $\mathrm{A}$ and B. In practice it is very useful to express all quantities in terms of such Euclidean notions of distance and direction as $R_{\mathrm{AB}}$ and $\boldsymbol{N}_{\mathrm{AB}}$. Once we have fixed our coordinate system to be $(t, \boldsymbol{x})$, we can forget about the curved geometry (A.1) and reason as if we were in flat space-time.

Clearly, with the two points $\mathrm{A}$ and $\mathrm{B}$ being given, the trajectory is entirely determined. In particular, the radial coordinate $r_{0}$ of the periastron is uniquely fixed as a function of $r_{\mathrm{A}}, r_{\mathrm{B}}$ and $R_{\mathrm{AB}}$. Neglecting terms in $1 / c^{2}$, we find $r_{0}^{2}=\frac{1}{4 R_{\mathrm{AB}}^{2}}\left(R_{\mathrm{AB}}^{2}-\left(r_{\mathrm{A}}-r_{\mathrm{B}}\right)^{2}\right)\left(\left(r_{\mathrm{A}}+r_{\mathrm{B}}\right)^{2}-R_{\mathrm{AB}}^{2}\right)$.

The unit vector $\boldsymbol{N}_{\mathrm{AB}}$ is given as

$\boldsymbol{N}_{\mathrm{AB}}=\boldsymbol{e}_{\alpha_{0}}-\frac{2 G M}{c^{2} r_{0}} \frac{r_{\mathrm{B}}-r_{\mathrm{A}}}{R_{\mathrm{AB}}} \boldsymbol{e}_{r_{0}}$.

On the other hand, the unit tangent to the trajectory at the emission point A (say) is given from (A.25) as

$\boldsymbol{n}_{\mathrm{A}}=\boldsymbol{e}_{\alpha_{0}}-\frac{2 G M}{c^{2} r_{0}} \sin \left(\alpha_{\mathrm{A}}-\alpha_{0}\right) \boldsymbol{e}_{r_{0}}$.

The difference between the two vectors (A.31) and (A.32) is a small quantity $\mathcal{O}\left(1 / c^{2}\right)$. It is not difficult, with the help of our solution for the trajectory [cf. (A.23)-(A.25) and (A.30)], to obtain the relation between these two vectors (always working consistently to the order $1 / c^{2}$ ):

$$
\begin{aligned}
\boldsymbol{n}_{\mathrm{A}} & =\boldsymbol{N}_{\mathrm{AB}} \\
& +\frac{4 G M}{c^{2} r_{\mathrm{A}}} \frac{R_{\mathrm{AB}}}{\left(r_{\mathrm{A}}+r_{\mathrm{B}}\right)^{2}-R_{\mathrm{AB}}^{2}}\left(\boldsymbol{r}_{\mathrm{A}}-\left(\boldsymbol{N}_{\mathrm{AB}} \cdot \boldsymbol{r}_{\mathrm{A}}\right) \boldsymbol{N}_{\mathrm{AB}}\right) .
\end{aligned}
$$


The coordinate time transfer from $\mathrm{A}$ to $\mathrm{B}$, denoted $T_{\mathrm{AB}}=$ $t_{\mathrm{B}}-t_{\mathrm{A}}$, follows from the Eq. (A.27). By expressing it with the help of the previous notation, notably of the "straight line" distance $R_{\mathrm{AB}}$, we obtain the simple formula

$T_{\mathrm{AB}}=\frac{R_{\mathrm{AB}}}{c}+\frac{2 G M}{c^{3}} \ln \left(\frac{r_{\mathrm{A}}+r_{\mathrm{B}}+R_{\mathrm{AB}}}{r_{\mathrm{A}}+r_{\mathrm{B}}-R_{\mathrm{AB}}}\right)$.

The second term, purely of order $1 / c^{3}$, is the Shapiro logarithmic time delay.

The right side of (A.34) depends only on the spatial positions of the emission and reception points, i.e. $\boldsymbol{r}_{\mathrm{A}}$ and $\boldsymbol{r}_{\mathrm{B}}$, and not on the instants $t_{\mathrm{A}}$ and/or $t_{\mathrm{B}}$. Indeed, for stationary space-times, the time $t-t_{\mathrm{A}}$ elapsed from some emission instant $t_{\mathrm{A}}$ does not depend on $t_{\mathrm{A}}$ but only on the spatial coordinates $\boldsymbol{r}_{\mathrm{A}}$ and $\boldsymbol{r}$, so there exists a function $V$ such that $c\left(t-t_{\mathrm{A}}\right)=V\left(\boldsymbol{r}_{\mathrm{A}}, \boldsymbol{r}\right)$. This function is the so-called optical point characteristic of the stationary space-time, or optical distance between pairs of points (Buchdahl 1970, 1979). In our case, we have

$V\left(\boldsymbol{r}_{\mathrm{A}}, \boldsymbol{r}\right)=R_{\mathrm{A}}+\frac{2 G M}{c^{2}} \ln \left(\frac{r_{\mathrm{A}}+r+R_{\mathrm{A}}}{r_{\mathrm{A}}+r-R_{\mathrm{A}}}\right)$,

where $\boldsymbol{R}_{\mathrm{A}}=\boldsymbol{r}-\boldsymbol{r}_{\mathrm{A}}, R_{\mathrm{A}}=\left|\boldsymbol{R}_{\mathrm{A}}\right|$. Note that along the light ray from $\boldsymbol{r}_{\mathrm{A}}$ to $\boldsymbol{r}$, the function $S$ defined by $S(t, \boldsymbol{r})=$ $-c t+V\left(\boldsymbol{r}_{\mathrm{A}}, \boldsymbol{r}\right)$ stays constant $\left(S=-c t_{\mathrm{A}}\right)$. Therefore $S$ represents the phase of the signal and can be used to define the wave-vector as $k_{\mu}=\partial_{\mu} S$. For this choice $k_{0}=-1$ and $k_{i}=\partial_{i} V$. Using (A.35) we get

$\frac{k_{i}}{k_{0}}=-N_{\mathrm{A}}^{i}-\frac{4 G M}{c^{2}} \frac{\left(r_{\mathrm{A}}+r\right) N_{\mathrm{A}}^{i}-R_{\mathrm{A}} \frac{r^{i}}{r}}{\left(r_{\mathrm{A}}+r\right)^{2}-R_{\mathrm{A}}^{2}}$,

where $N_{\mathrm{A}}^{i}=R_{\mathrm{A}}^{i} / R_{\mathrm{A}}$. This is in complete agreement with (A.18) and (A.33).

Given the simplicity of the result (A.34) for the time transfer when expressed in terms of the Euclidean distance $R_{\mathrm{AB}}$, one can guess that the formula can be derived directly by integrating $\mathrm{d} s^{2}=0$ along the path from A to B. Let us show how this works. With the post-Newtonian metric (A.1)-(A.3) we have, along the photon's path,

$c \mathrm{~d} t=\left(1+\frac{2 G M}{r c^{2}}\right)|\mathrm{d} \boldsymbol{r}|$,

where $|\mathrm{d} \boldsymbol{r}|$ is the Euclidean norm of the vector $\mathrm{d} \boldsymbol{r}=\mathrm{d} \boldsymbol{x}$. Introducing $\boldsymbol{R}_{\mathrm{A}}=\boldsymbol{r}-\boldsymbol{r}_{\mathrm{A}}$ as a new spatial coordinate along the path, we have $|\mathrm{d} \boldsymbol{r}|=\left(\mathrm{d} R_{\mathrm{A}}^{2}+R_{\mathrm{A}}^{2} \mathrm{~d} \boldsymbol{N}_{\mathrm{A}}^{2}\right)^{1 / 2}$ where $R_{\mathrm{A}}=\left|\boldsymbol{R}_{\mathrm{A}}\right|$ and $\boldsymbol{N}_{\mathrm{A}}=\boldsymbol{R}_{\mathrm{A}} / R_{\mathrm{A}}$. But we know from (A.33) that $\boldsymbol{N}_{\mathrm{A}}$ differs from the unit tangent $\boldsymbol{n}_{\mathrm{A}}$ at the emission point by a small term $\mathcal{O}\left(1 / c^{2}\right)$. So, when $\boldsymbol{r}$ varies (the origin point $\mathrm{A}$ on the path being fixed), we have $\mathrm{d} \boldsymbol{N}_{\mathrm{A}}=$ $\mathcal{O}\left(1 / c^{2}\right)$ and therefore we see that $\mathrm{d} \boldsymbol{N}_{\mathrm{A}}^{2}=\mathcal{O}\left(1 / c^{4}\right)$ makes a negligible contribution. This shows that to order $1 / c^{2}$ inclusively the time transfer can be calculated "along the straight line"; we have

$\mathrm{d} t=\left(1+\frac{2 G M}{r c^{2}}\right) \frac{\mathrm{d} R_{\mathrm{A}}}{c}$, and the total time transfer reads

$T_{\mathrm{AB}}=\frac{R_{\mathrm{AB}}}{c}+\frac{2 G M}{c^{3}} \int_{0}^{R_{\mathrm{AB}}} \frac{\mathrm{d} R_{\mathrm{A}}}{r}$.

To find the closed-form expression of the integral, we insert $r=\left(R_{\mathrm{A}}^{2}+2 \boldsymbol{R}_{\mathrm{A}} \cdot \boldsymbol{r}_{\mathrm{A}}+r_{\mathrm{A}}^{2}\right)^{1 / 2}$ which can be approximated, since $\boldsymbol{N}_{\mathrm{A}}=\boldsymbol{n}_{\mathrm{A}}+\mathcal{O}\left(1 / c^{2}\right)$ and the integral already enters a small quantity, by $r=\left(R_{\mathrm{A}}^{2}+2 R_{\mathrm{A}} \boldsymbol{n}_{\mathrm{A}} \cdot \boldsymbol{r}_{\mathrm{A}}+r_{\mathrm{A}}^{2}\right)^{1 / 2}$. Next we perform the integration over $R_{\mathrm{A}}$ from $\mathrm{A}$ to $\mathrm{B}$, and we are allowed to replace $\boldsymbol{n}_{\mathrm{A}}=\boldsymbol{N}_{\mathrm{AB}}+\mathcal{O}\left(1 / c^{2}\right)$ within the result. Finally we find (see e.g. Will 1981)

$T_{\mathrm{AB}}=\frac{R_{\mathrm{AB}}}{c}+\frac{2 G M}{c^{3}} \ln \left(\frac{r_{\mathrm{B}}+\boldsymbol{r}_{\mathrm{B}} \cdot \boldsymbol{N}_{\mathrm{AB}}}{r_{\mathrm{A}}+\boldsymbol{r}_{\mathrm{A}} \cdot \boldsymbol{N}_{\mathrm{AB}}}\right)$.

This expression for the Shapiro time delay is slightly different from the previous form (A.34) but can easily be reconciled with it. To this end one makes use of the identities

$r_{\mathrm{B}}+\boldsymbol{r}_{\mathrm{B}} \cdot \boldsymbol{N}_{\mathrm{AB}}=\frac{\left(r_{\mathrm{B}}+R_{\mathrm{AB}}\right)^{2}-r_{\mathrm{A}}^{2}}{2 R_{\mathrm{AB}}}$,
$r_{\mathrm{A}}+\boldsymbol{r}_{\mathrm{A}} \cdot \boldsymbol{N}_{\mathrm{AB}}=\frac{r_{\mathrm{B}}^{2}-\left(r_{\mathrm{A}}-R_{\mathrm{AB}}\right)^{2}}{2 R_{\mathrm{AB}}}$,

which show that (A.34) and (A.40) are indeed totally equivalent. However, in practice, we shall prefer to use the formula (A.34) rather than (A.40) because of its structural simplicity.

Sagnac terms. The formula (A.34) gives the time transfer from the point $\boldsymbol{r}_{\mathrm{A}} \equiv \boldsymbol{r}_{\mathrm{A}}\left(t_{\mathrm{A}}\right)$ at the emission instant $t_{\mathrm{A}}$, to the reception point $\boldsymbol{r}_{\mathrm{B}} \equiv \boldsymbol{r}_{\mathrm{B}}\left(t_{\mathrm{B}}\right)$ at the reception instant $t_{\mathrm{B}}$. But in fact, in the application to ACES, the useful time basis in the experiment is that provided by the clock at the point $\mathrm{A}$ (i.e., in the satellite) which records the instant of emission $t_{\mathrm{A}}$. Therefore, it is more convenient to re-express the time transfer (A.34) in terms of the position of the reception point $\mathrm{B}$ as it was at the instant $t_{\mathrm{A}}$ rather than at $t_{\mathrm{B}}$, i.e. when it was instantaneous with the instant of emission at A (in the GRS coordinate system). Thus, we introduce the "instantaneous" coordinate distance

$\boldsymbol{D}_{\mathrm{AB}}=\boldsymbol{r}_{\mathrm{B}}\left(t_{\mathrm{A}}\right)-\boldsymbol{r}_{\mathrm{A}}\left(t_{\mathrm{A}}\right) ; \quad \boldsymbol{D}_{\mathrm{AB}}=\left|\boldsymbol{D}_{\mathrm{AB}}\right|$,

and perform a consistent series expansion when $1 / c$ tends to zero. We know from (A.34) that the time transfer $T_{\mathrm{AB}}=\mathcal{O}(1 / c)$. Therefore, with the required accuracy, we can write $\boldsymbol{R}_{\mathrm{AB}}=\boldsymbol{D}_{\mathrm{AB}}+T_{\mathrm{AB}} \boldsymbol{v}_{\mathrm{B}}\left(t_{\mathrm{A}}\right)+\frac{1}{2} T_{\mathrm{AB}}^{2} \boldsymbol{a}_{\mathrm{B}}\left(t_{\mathrm{A}}\right)+$ $\mathcal{O}\left(1 / c^{3}\right)$, where $\boldsymbol{a}_{\mathrm{B}}$ is the acceleration. The successive relativistic approximations are obtained by working out this formula iteratively together with (A.34). In this way we obtain

$$
\begin{aligned}
T_{\mathrm{AB}} & =\frac{D_{\mathrm{AB}}}{c}+\frac{\boldsymbol{D}_{\mathrm{AB}} \cdot \boldsymbol{v}_{\mathrm{B}}\left(t_{\mathrm{A}}\right)}{c^{2}} \\
& +\frac{D_{\mathrm{AB}}}{2 c^{3}}\left(\boldsymbol{v}_{\mathrm{B}}^{2}+\frac{\left(\boldsymbol{D}_{\mathrm{AB}} \cdot \boldsymbol{v}_{\mathrm{B}}\right)^{2}}{\boldsymbol{D}_{\mathrm{AB}}^{2}}+\boldsymbol{D}_{\mathrm{AB}} \cdot \boldsymbol{a}_{\mathrm{B}}\right) \\
& +\frac{2 G M}{c^{3}} \ln \left(\frac{r_{\mathrm{A}}+r_{\mathrm{B}}+D_{\mathrm{AB}}}{r_{\mathrm{A}}+r_{\mathrm{B}}-D_{\mathrm{AB}}}\right)
\end{aligned}
$$


where all quantities are measured at the emission instant $t_{\mathrm{A}}$ recorded by the on-board clock. The formula involves the Sagnac terms of first $\left(1 / c^{2}\right)$ and second $\left(1 / c^{3}\right)$ orders, as well as the Shapiro time delay [of course, consistently with the approximation, one can use indifferently in the Shapiro term either the distance $D_{\mathrm{AB}}$ or $R_{\mathrm{AB}}$, and either $r_{\mathrm{B}}\left(t_{\mathrm{B}}\right)$ or $\left.r_{\mathrm{B}}\left(t_{\mathrm{A}}\right)\right]$.

One-way frequency transfer. In the geometric optics approximation we have $k_{\mu}=\partial_{\mu} S$ and the phase difference between two successive electromagnetic pulses is $\mathrm{d} S=$ $\left(\partial_{\mu} S \mathrm{~d} x^{\mu}\right)_{\mathrm{A}}=\left(\partial_{\mu} S \mathrm{~d} x^{\mu}\right)_{\mathrm{B}}$, which in other words means $\left(k_{\mu} u^{\mu} \mathrm{d} \tau\right)_{\mathrm{A}}=\left(k_{\mu} u^{\mu} \mathrm{d} \tau\right)_{\mathrm{B}}$ where $u^{\mu}=\mathrm{d} x^{\mu} / \mathrm{d} \tau$ denotes the four-velocity (such that $g_{\mu \nu} u^{\mu} u^{\nu}=-1$ ). Now, $\mathrm{d} \tau_{\mathrm{A}}$ and $\mathrm{d} \tau_{\mathrm{B}}$ are the proper periods of the same signal at $\mathrm{A}$ and $\mathrm{B}$ and we have $\nu_{\mathrm{A}} / \nu_{\mathrm{B}}=\mathrm{d} \tau_{\mathrm{B}} / \mathrm{d} \tau_{\mathrm{A}}$. Therefore, the one-way frequency shift of photons during the transfer from $\mathrm{A}$ to $\mathrm{B}$ is

$$
\frac{\nu_{\mathrm{A}}}{\nu_{\mathrm{B}}}=\frac{\left(k_{\mu} u^{\mu}\right)_{\mathrm{A}}}{\left(k_{\mu} u^{\mu}\right)_{\mathrm{B}}}
$$

Separating out temporal from spatial components, we have $k_{\mu} u^{\mu}=u^{0}\left(k_{0}+k_{i} v^{i} / c\right)$, where $v^{i}=c u^{i} / u^{0}$ denotes the coordinate velocity and where $u^{0}=$ $\left(-g_{\rho \sigma} v^{\rho} v^{\sigma} / c^{2}\right)^{-1 / 2}$. In a stationary space-time $k_{0}$ is constant along the trajectory, i.e. $\left(k_{0}\right)_{\mathrm{A}}=\left(k_{0}\right)_{\mathrm{B}}=-\hat{E} / c$ in the notation (A.6); for instance, we have seen that with the choice $S(t, \boldsymbol{r})=-c t+V\left(\boldsymbol{r}_{\mathrm{A}}, \boldsymbol{r}\right)$, where $V$ is the optical distance (A.35), this constant is equal to minus one. Thus we can write

$\frac{\nu_{\mathrm{A}}}{\nu_{\mathrm{B}}}=\frac{\left(u^{0}\right)_{\mathrm{A}}}{\left(u^{0}\right)_{\mathrm{B}}} \frac{1+\left(\frac{k_{i}}{k_{0}}\right)_{\mathrm{A}} \frac{v_{\mathrm{A}}^{i}}{c}}{1+\left(\frac{k_{i}}{k_{0}}\right)_{\mathrm{B}} \frac{v_{\mathrm{B}}^{i}}{c}}$.

Next, we found in (A.18) that the covariant components of the wave vector $k_{i}$ are proportional to the unit tangent vector $n^{i}$. Using this we can further infer that

$\frac{\nu_{\mathrm{A}}}{\nu_{\mathrm{B}}}=\frac{\left(u^{0}\right)_{\mathrm{A}}}{\left(u^{0}\right)_{\mathrm{B}}} \frac{1-\sqrt{\frac{g\left(r_{\mathrm{A}}\right)}{f\left(r_{\mathrm{A}}\right)}} \frac{\boldsymbol{n}_{\mathrm{A}} \cdot \boldsymbol{v}_{\mathrm{A}}}{c}}{1-\sqrt{\frac{g\left(r_{\mathrm{B}}\right)}{f\left(r_{\mathrm{B}}\right)}} \frac{\boldsymbol{n}_{\mathrm{B}} \cdot \boldsymbol{v}_{\mathrm{B}}}{c}}$.

Within the first factor $u^{0}$ is the coordinate time $v s$ proper time ratio, which has been computed in Eq. (3). Thus, from $\left(u^{0}\right)_{\mathrm{A}} /\left(u^{0}\right)_{\mathrm{B}}=(\mathrm{d} \tau / \mathrm{d} t)_{\mathrm{B}} /(\mathrm{d} \tau / \mathrm{d} t)_{\mathrm{A}}$, we get

$$
\frac{\left(u^{0}\right)_{\mathrm{A}}}{\left(u^{0}\right)_{\mathrm{B}}}=\frac{1-\frac{1}{c^{2}}\left[U_{\mathrm{E}}\left(\boldsymbol{r}_{\mathrm{B}}\right)+\frac{\boldsymbol{v}_{\mathrm{B}}^{2}}{2}\right]}{1-\frac{1}{c^{2}}\left[U_{\mathrm{E}}\left(\boldsymbol{r}_{\mathrm{A}}\right)+\frac{\boldsymbol{v}_{\mathrm{A}}^{2}}{2}\right]},
$$

where $U_{\mathrm{A}}$ and $U_{\mathrm{B}}$ are the Newtonian potentials at the levels A and B. The factor (A.48) comprises the Einstein gravitational red-shift and the second-order Doppler effects, which are both of order $1 / c^{2}$. We recall from Sect. 2 that at the level of accuracy of ACES, the potentials in (A.48) must take into account the oblaticity of the Earth.

Now the second factor in the right-hand-side of (A.47) is nothing but the ratio of coordinate periods of the same signal at $\mathrm{A}$ and $\mathrm{B}$, namely $\mathrm{d} t_{\mathrm{B}} / \mathrm{d} t_{\mathrm{A}}$; it contains the firstorder Doppler effect $(\sim 1 / c)$ and the third-order $\left(\sim 1 / c^{3}\right)$ terms we are looking for. For the computation of this factor at the level $510^{-17}$ we do not need to consider the $J_{2}$ of the Earth potential, and we can approximate $U_{\mathrm{E}}=G M / r$. In order to obtain a useful formula, we have only to substitute for the tangent vectors $\boldsymbol{n}_{\mathrm{A}}$ and $\boldsymbol{n}_{\mathrm{B}}$ their expressions in terms of the unit direction $\boldsymbol{N}_{\mathrm{AB}}$ into (A.47). The required relation between these vectors was found in (A.33). Alternatively, one can use directly the formula (A.36). Finally, our end-result for the one-way frequency transfer with $1 / c^{3}$ accuracy reads

$\frac{\nu_{\mathrm{A}}}{\nu_{\mathrm{B}}}=\frac{\left(u^{0}\right)_{\mathrm{A}}}{\left(u^{0}\right)_{\mathrm{B}}} \frac{q_{\mathrm{A}}}{q_{\mathrm{B}}}$,

where the ratio $q_{\mathrm{A}} / q_{\mathrm{B}}$ is given by the formulas (14)-(15) in the text.

The previous expressions were obtained using our explicit solution (A.23)-(A.25) for the photon's trajectory. However, there is a simpler way to obtain the ratio $q_{\mathrm{A}} / q_{\mathrm{B}}$, which is based on the fact that since it is equal to the ratio of coordinate times: $q_{\mathrm{A}} / q_{\mathrm{B}}=\mathrm{d} t_{\mathrm{B}} / \mathrm{d} t_{\mathrm{A}}$, it can be computed directly by differentiating the coordinate time transfer $T_{\mathrm{AB}}=t_{\mathrm{B}}-t_{\mathrm{A}}$ with respect to the emission time $t_{\mathrm{A}}$. The time transfer to order $1 / c^{3}$ is given by the simple formula (A.34) containing the Shapiro term. Thus, we must consider

$$
\begin{aligned}
& \frac{\mathrm{d}}{\mathrm{d} t_{\mathrm{A}}}\left(t_{\mathrm{B}}-t_{\mathrm{A}}\right)= \\
& \quad \frac{\mathrm{d}}{\mathrm{d} t_{\mathrm{A}}}\left\{\frac{R_{\mathrm{AB}}}{c}+\frac{2 G M}{c^{3}} \ln \left(\frac{r_{\mathrm{A}}+r_{\mathrm{B}}+R_{\mathrm{AB}}}{r_{\mathrm{A}}+r_{\mathrm{B}}-R_{\mathrm{AB}}}\right)\right\} .
\end{aligned}
$$

The differentiation is to be performed taking into account the fact that the coordinate distance between A and B depends on both the emission and reception times, i.e. $R_{\mathrm{AB}}=\left|\boldsymbol{r}_{\mathrm{B}}\left(t_{B}\right)-\boldsymbol{r}_{\mathrm{A}}\left(t_{\mathrm{A}}\right)\right|$. Thus we have for instance

$\frac{\mathrm{d} R_{\mathrm{AB}}}{\mathrm{d} t_{\mathrm{A}}}=\boldsymbol{N}_{\mathrm{AB}} \cdot\left(\boldsymbol{v}_{\mathrm{B}} \frac{\mathrm{d} t_{\mathrm{B}}}{\mathrm{d} t_{\mathrm{A}}}-\boldsymbol{v}_{\mathrm{A}}\right)$.

We find that the ratio $q_{\mathrm{A}} / q_{\mathrm{B}}=\mathrm{d} t_{\mathrm{B}} / \mathrm{d} t_{\mathrm{A}}$ as obtained from the Eq. (A.50) agrees exactly with the one given by the expressions (14)-(15).

Two-way frequency transfer. In this method the "tracking" signal is emitted from the ground station at point $\mathrm{B}^{\prime}$ and coordinate time $t_{\mathrm{B}^{\prime}}$, is received by the satellite at point $\mathrm{A}$ and time $t_{\mathrm{A}}$ and immediately transponded back to the ground station where it arrives at point $\mathrm{B}$ and time $t_{\mathrm{B}}$. (A generalization of the formulae to the case where there is a time delay between reception and re-emission at $\mathrm{A}$ is also possible.) The "clock" signal is emitted at A, simultaneously with the tracking signal when it is transponded at $\mathrm{A}$, and transmitted to $\mathrm{B}$. This technique, called the Doppler-cancelling technique, permits us to suppress the first-order Doppler effect and to drastically limit the uncertainties linked with the atmospheric contributions (see 
e.g. Kleppner et al. 1970). The ratio of the signal frequencies to be inserted in (12) follows from

$\frac{\nu_{\mathrm{B}}}{\nu_{\mathrm{A}}}=\frac{1}{2}\left(\frac{\nu_{\mathrm{B}}}{\nu_{\mathrm{B}^{\prime}}}\right)_{\text {station }}+\Delta_{\mathrm{AB}}+\frac{1}{2}$,

where $\left(\nu_{\mathrm{B}} / \nu_{\mathrm{B}^{\prime}}\right)_{\text {station }}$ is measured at the ground station, and where $\Delta_{\mathrm{AB}}$ is given theoretically by

$\Delta_{\mathrm{AB}}=\left(\frac{\nu_{\mathrm{B}}}{\nu_{\mathrm{A}}}\right)\left[1-\frac{1}{2}\left(\frac{\nu_{\mathrm{A}}}{\nu_{\mathrm{B}^{\prime}}}\right)\right]-\frac{1}{2}$.

The ratios $\nu_{\mathrm{B}} / \nu_{\mathrm{A}}$ and $\nu_{\mathrm{A}} / \nu_{\mathrm{B}^{\prime}}$ are given by the formulas (13)-(15), valid for the one-way transfer, that we must simply insert into (A.53) in order to get the result. The only problem is to correctly express all the quantities associated with the up-link from the ground station $\mathrm{B}^{\prime}$ at $t_{\mathrm{B}^{\prime}}$ to the satellite $\mathrm{A}$ at $t_{\mathrm{A}}$, in terms of the same quantities associated with the down-link back from the satellite A to the station $\mathrm{B}$ at $t_{\mathrm{B}}$ (see Fig. 2). Notably we need to express the unit vector $\boldsymbol{N}_{\mathrm{B}^{\prime} \mathrm{A}}$ in terms of the unit vector $\boldsymbol{N}_{\mathrm{AB}}$ on the way back, as well as the velocity and acceleration $\boldsymbol{v}_{\mathrm{B}}\left(t_{\mathrm{B}}\right)$ and $\boldsymbol{a}_{\mathrm{B}}\left(t_{\mathrm{B}}\right)$ of the ground station at $t_{\mathrm{B}}$. Neglecting $\mathcal{O}\left(1 / c^{3}\right)$ we find

$$
\begin{aligned}
\boldsymbol{N}_{\mathrm{B}^{\prime} \mathrm{A}}= & -\boldsymbol{N}_{\mathrm{AB}}\left\{1+\frac{2}{c} \boldsymbol{N}_{\mathrm{AB}} \cdot \boldsymbol{v}_{\mathrm{B}}\right. \\
& \left.+\frac{1}{c^{2}}\left[4\left(\boldsymbol{N}_{\mathrm{AB}} \cdot \boldsymbol{v}_{\mathrm{B}}\right)^{2}-2 \boldsymbol{v}_{\mathrm{B}}^{2}-2 \boldsymbol{R}_{\mathrm{AB}} \cdot \boldsymbol{a}_{\mathrm{B}}\right]\right\} \\
& +\frac{2}{c} \boldsymbol{v}_{\mathrm{B}}\left(1+\frac{1}{c} \boldsymbol{N}_{\mathrm{AB}} \cdot \boldsymbol{v}_{\mathrm{B}}\right)-\frac{2}{c^{2}} R_{\mathrm{AB}} \boldsymbol{a}_{\mathrm{B}} .
\end{aligned}
$$

We need also the velocity of the station at emission, $\boldsymbol{v}_{\mathrm{B}^{\prime}}\left(t_{\mathrm{B}^{\prime}}\right)$, in terms of its velocity, acceleration and derivative of acceleration at reception, $\boldsymbol{v}_{\mathrm{B}}\left(t_{\mathrm{B}}\right), \boldsymbol{a}_{\mathrm{B}}\left(t_{\mathrm{B}}\right)$ and $\boldsymbol{b}_{\mathrm{B}}\left(t_{\mathrm{B}}\right)$. We get

$\boldsymbol{v}_{\mathrm{B}^{\prime}}=\boldsymbol{v}_{\mathrm{B}}-\frac{2}{c} R_{\mathrm{AB}} \boldsymbol{a}_{\mathrm{B}}+\frac{2}{c^{2}}\left[\left(\boldsymbol{R}_{\mathrm{AB}} \cdot \boldsymbol{v}_{\mathrm{B}}\right) \boldsymbol{a}_{\mathrm{B}}+R_{\mathrm{AB}}^{2} \boldsymbol{b}_{\mathrm{B}}\right]$.

These relativistic expansions being fully taken into account, we obtain finally, after a long computation, the formula (17) in the text, which is

$$
\begin{aligned}
\Delta_{\mathrm{AB}} & =\frac{1}{c^{2}}\left[U_{\mathrm{AB}}-\frac{1}{2} \boldsymbol{v}_{\mathrm{AB}}^{2}-\boldsymbol{R}_{\mathrm{AB}} \cdot \boldsymbol{a}_{\mathrm{B}}\right]\left(1+\frac{\boldsymbol{N}_{\mathrm{AB}} \cdot \boldsymbol{v}_{\mathrm{AB}}}{c}\right) \\
& +\frac{R_{\mathrm{AB}}}{c^{3}}\left(-\boldsymbol{v}_{\mathrm{A}} \cdot \boldsymbol{a}_{\mathrm{B}}+\boldsymbol{R}_{\mathrm{AB}} \cdot \boldsymbol{b}_{\mathrm{B}}+2 \boldsymbol{v}_{\mathrm{B}} \cdot \boldsymbol{a}_{\mathrm{B}}-\boldsymbol{v}_{\mathrm{B}} \cdot \nabla U_{\mathrm{B}}\right),
\end{aligned}
$$

where $U_{\mathrm{AB}}=U_{\mathrm{B}}-U_{\mathrm{A}}$ and $\boldsymbol{v}_{\mathrm{AB}}=\boldsymbol{v}_{\mathrm{A}}-\boldsymbol{v}_{\mathrm{B}}$. All the quantities at $\mathrm{A}$ or $\mathrm{B}$ are expressed at the corresponding instants $t_{\mathrm{A}}$ or $t_{\mathrm{B}}$ respectively.

\section{References}

Allan, D. W., \& Ashby, N. 1985, in Relativity in Celestial Mechanics and Astronomy, Proc. of the IAU Symp., No. 114, Leningrad 1985, ed. J. Kovalevsky, \& V. A. Brumberg (Reidel, Dordrecht), 299

Ashby, N. 1998, IEEE International Frequency Control Symposium, 320

Ashby, N., \& Allan, D. W. 1979, Rad. Sci., 14(4), 649

Bize, S., et al. 1999, Europhys. Lett., 45(5), 558

Brumberg, V. A., \& Kopejkin, S. M. 1990, Celest. Mech. Dyn. Astron., 48, 23

Buchdahl, H. A. 1970, Optica Acta., 17, 707

Buchdahl, H. A. 1979, Aust. J. Phys., 32, 405

Fridelance, P., Samain, E., \& Veillet, C. 1996, Exper. Astron., 191

Hetzel, P., \& Soring, A. 1993, Proc. 7th European Frequency and Time Forum, Neuchâtel, 1993, Swiss Foundation for Research in Microtechnology, 125

IAU transactions 1991, vol. XXIB, Proc. 21st Gen. Assembly Buenos Aires (Kluwer, Dordrecht, Boston, London)

IAU 2000 transactions, in press; the complete resolutions can be found e.g. on http://danof .obspm.fr/iaucom19

Jaffe, J., \& Vessot, R. F. C. 1976, Phys. Rev. D, 14, 3294

Kleppner, D., Vessot, R. F. C., \& Ramsey, N. F. 1970, Astroph. Space Sci., 6, 13

Klioner, S. A. 1993, Celest. Mech. Dyn. Astron., 53, 81

Klioner, S. A., \& Fukushima, T. 1994, Manuscr. Geod., 19, 294

Kopeikin, S. M., \& Schäfer, G. 1999, Phys. Rev. D, 60, 124002

Laurent, P., Lemonde P., Santarelli G., et al. 1999, in Proc. of the 14th Int. Conf. on Laser Spectrocopy, ed. R. Blatt, J. Eschner, D. Leibfried, \& F. Schmidt-Kaler (World Scientific)

Misner, C. W., Thorne, K. S., \& Wheeler, J. A. 1973, Gravitation (Freeman)

Petit, G., \& Wolf, P. 1994, A\&A, 286, 971

Petit, G., \& Wolf, P. 1997, IEEE Trans. Instrum. Meas., 46, 201

Salomon, C., \& Veillet, C. 1996, in Proc. Symp. on Space Station Utilisation, ESA-SP 385, 295

Shapiro, I. I. 1964, Phys. Rev. Lett., 13, 789

Veillet, C., \& Fridelance, P. 1993, Proc. 7th European Frequency and Time Forum, Neuchâtel, 1993, Swiss Foundation for Research in Microtechnology, 637

Vessot, R. F. C., Levine, M. W., et al. 1980, Phys. Rev. Lett., 45,2081

Will, C. M. 1981, Theory and Experiment in Gravitational Physics (Cambridge U. Press)

Wolf, P., \& Petit, G. 1995, A\&A, 304, 653 